\title{
Microaneurysms Detection in Color Fundus Images based on Naive Bayesian Classification
}

\section{CURRENT STATUS: UNDER REVISION}

BioMedical Engineering OnLine $\triangle B M C$

Shengchun Long

Zhejiang University of Technology

Jiali Chen

Zhejiang University of Technology

ح chenjiali@zjut.edu.cnCorresponding Author

ORCiD: https://orcid.org/0000-0003-4102-4335

\section{Ante $\mathrm{Hu}$}

Zhejiang University of Technology

Haipeng Liu

Coventry University

Zhiqing Chen

Zhejiang University School of Medicine Second Affiliated Hospital

Dingchang Zheng

Coventry University

\section{DOI:}

$10.21203 / \mathrm{rs} .2 .21517 / \mathrm{v} 1$

\section{SUBJECT AREAS}

Biomedical Engineering

KEYWORDS

color fundus image; microaneurysms detection; patch; features extraction; Naive Bayesian classier 
Abstract

Background: As a major complication of diabetes, diabetic retinopathy (DR) is a leading cause of visual impairment and blindness due to delayed diagnosis and intervention. Microaneurysm appears as the earliest symptom of DR. Accurate and reliable detection of microaneurysms in color fundus images has great importance for DR screening. Methods: A microaneurysms detection method based on Naive Bayesian classifier is proposed for the early diagnosis of DR. First, blood vessels were enhanced and segmented using the analysis of eigenvalues of Hessian matrix. Next, with blood vessels excluded, microaneurysm candidate regions were obtained according to shape characteristics. After image segmented to patches, the features of each microaneurysm candidate patch were extracted, and each candidate patch was classified using Naive Bayes into microaneurysm or non- microaneurysm. The proposed algorithm was trained and tested on e-ophtha MA database, and further tested on another independent DIARETDB1 database. Results of microaneurysms detection on the two databases were evaluated on lesion level and compared with existing algorithms. Results: The proposed method has achieved better performance compared with existing algorithms. On e-ophtha MA and DIARETDB1 databases, the area under curve (AUC) of receiver operating characteristic $(\mathrm{ROC})$ curve was 0.845 and 0.831 , respectively. The free-response ROC (FROC) score on the two databases was 0.362 and 0.207 , respectively. Conclusions: The proposed method based on Naive Bayesian classification of image patches can effectively detect microaneurysms in color fundus images and provide an effective scientific basis for early clinical DR diagnosis.

Full-text

Due to technical limitations, full-text HTML conversion of this manuscript could not be completed. However, the manuscript can be downloaded and accessed as a PDF.

Figures 


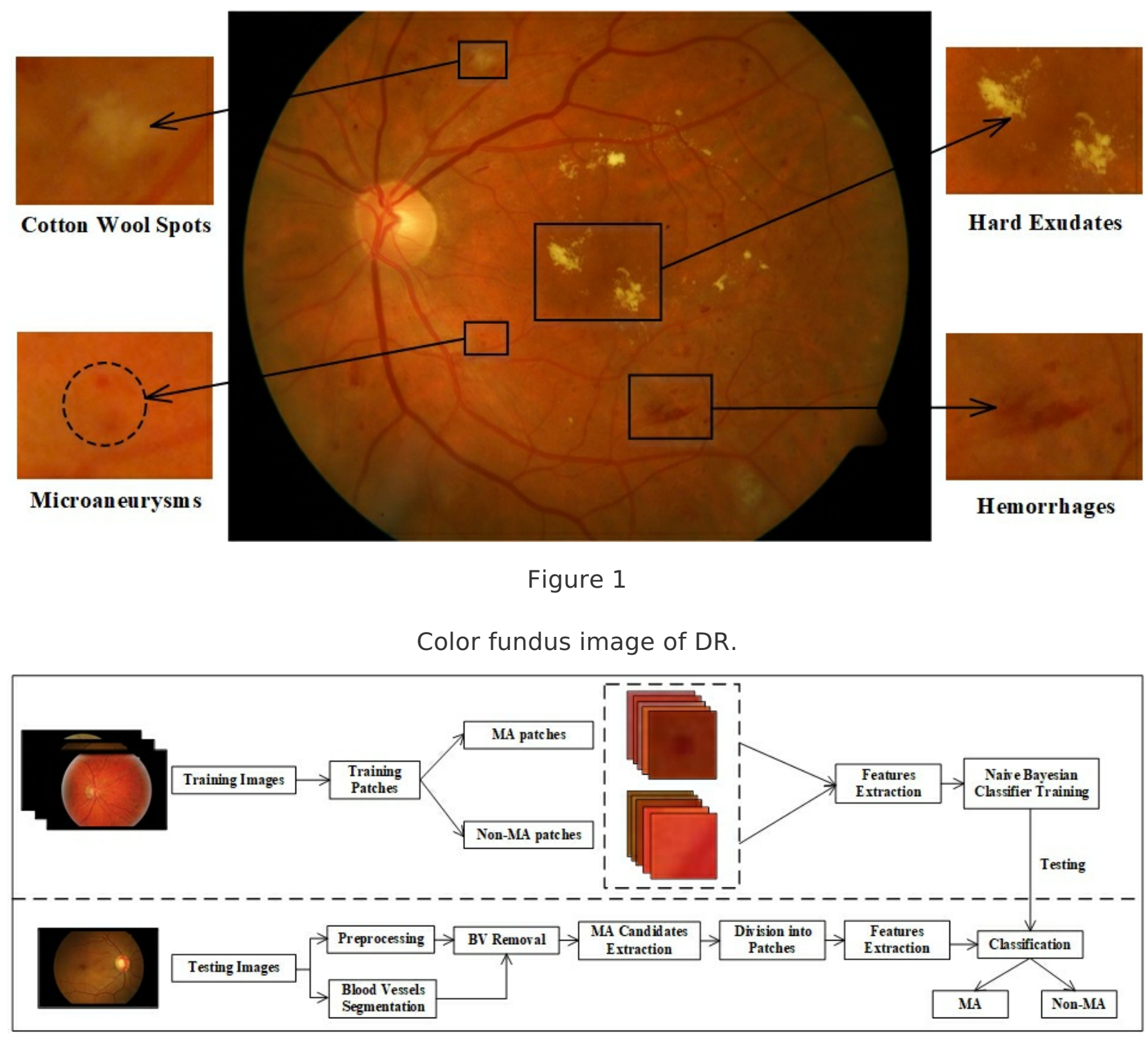

Figure 2

Illustration of the proposed MA detection method. 


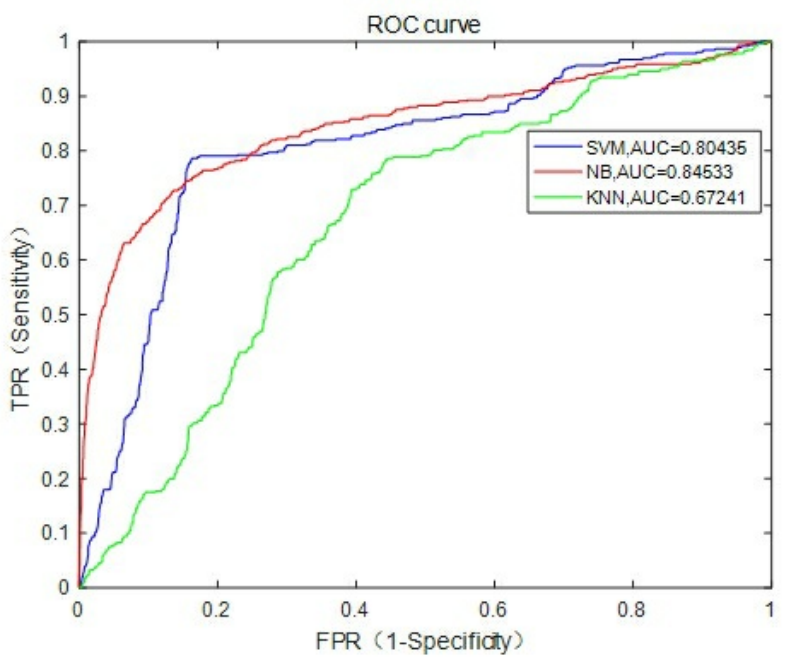

(a)

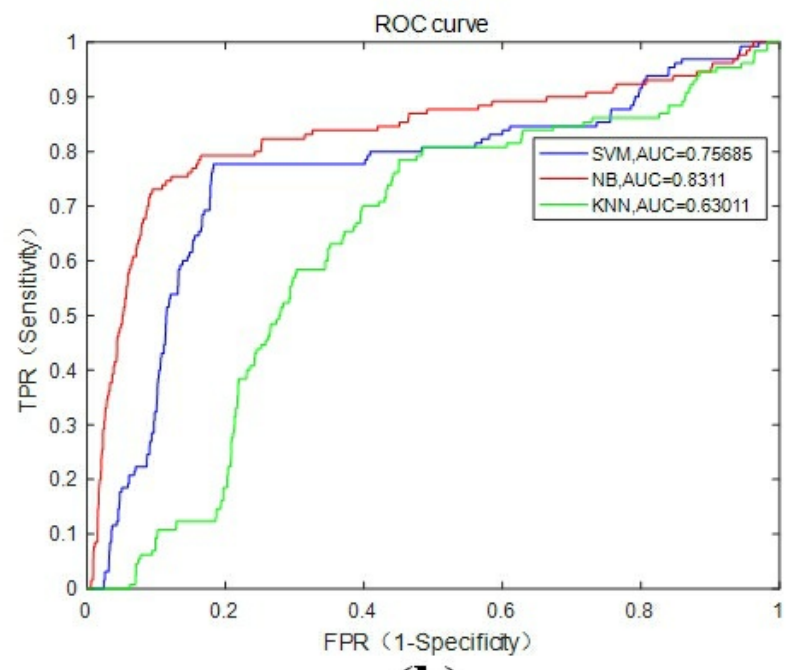

(b)

Figure 3

ROC curves of 3 classiers on the two databases. (a) e-ophtha MA database; (b) DIARETDB1 database

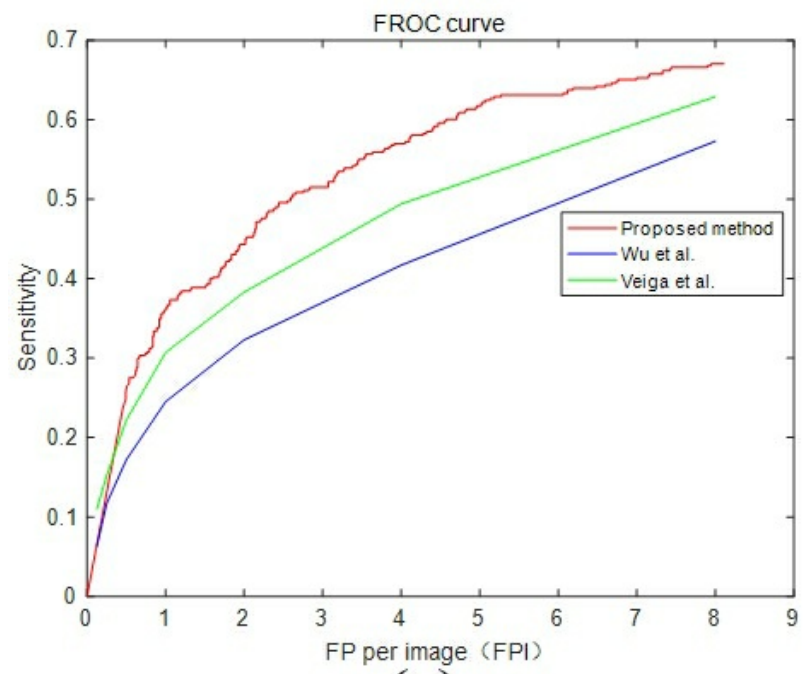

(a)

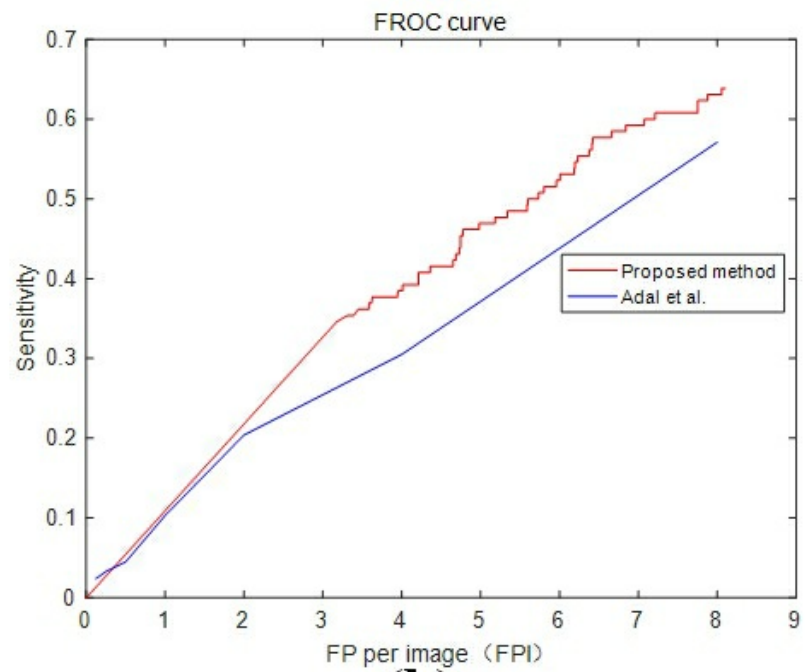

(b)

Figure 4

FROC curves of dierent methods for MA detection on the two databases. (a) e-ophtha MA database; (b) DIARETDB1 database 


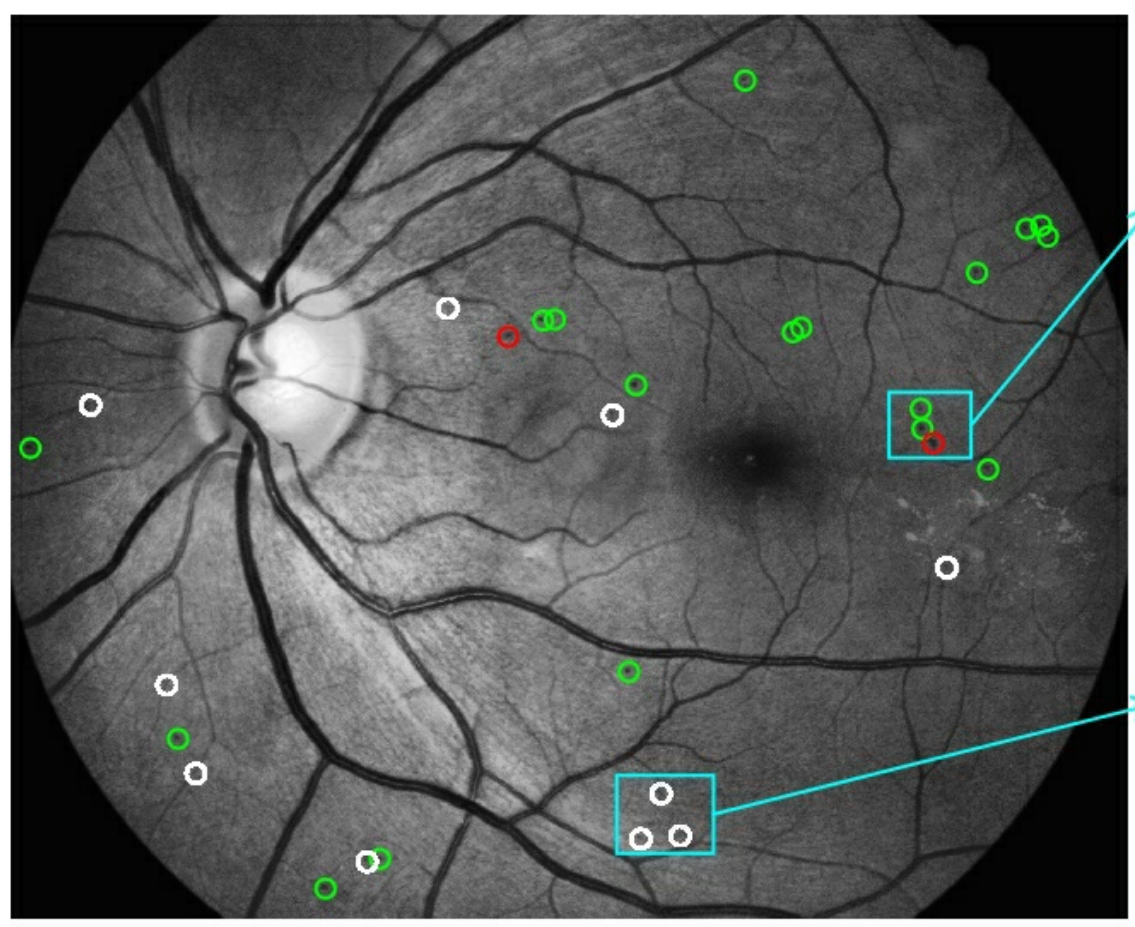

(a)

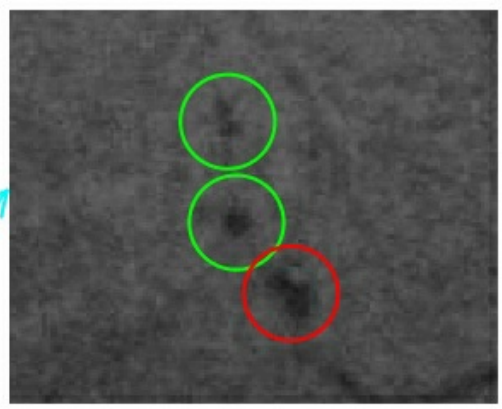

(b)

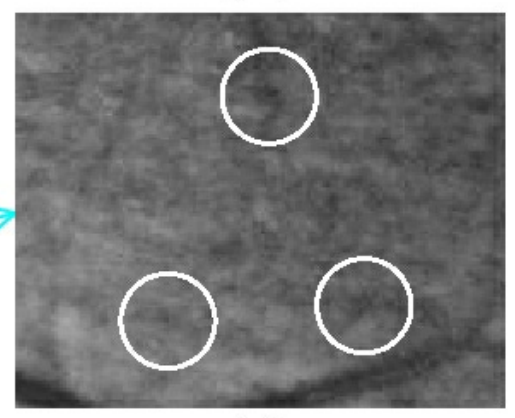

(c)

Figure 5

Lesion level evaluation for MA detection results on e-ophtha MA database. (a) Results of MA detection, where green circles indicate TPs, white circles indicate FPs, and red circles indicate FNs; (b) Examples of TP and FN; (c) Examples of FP 


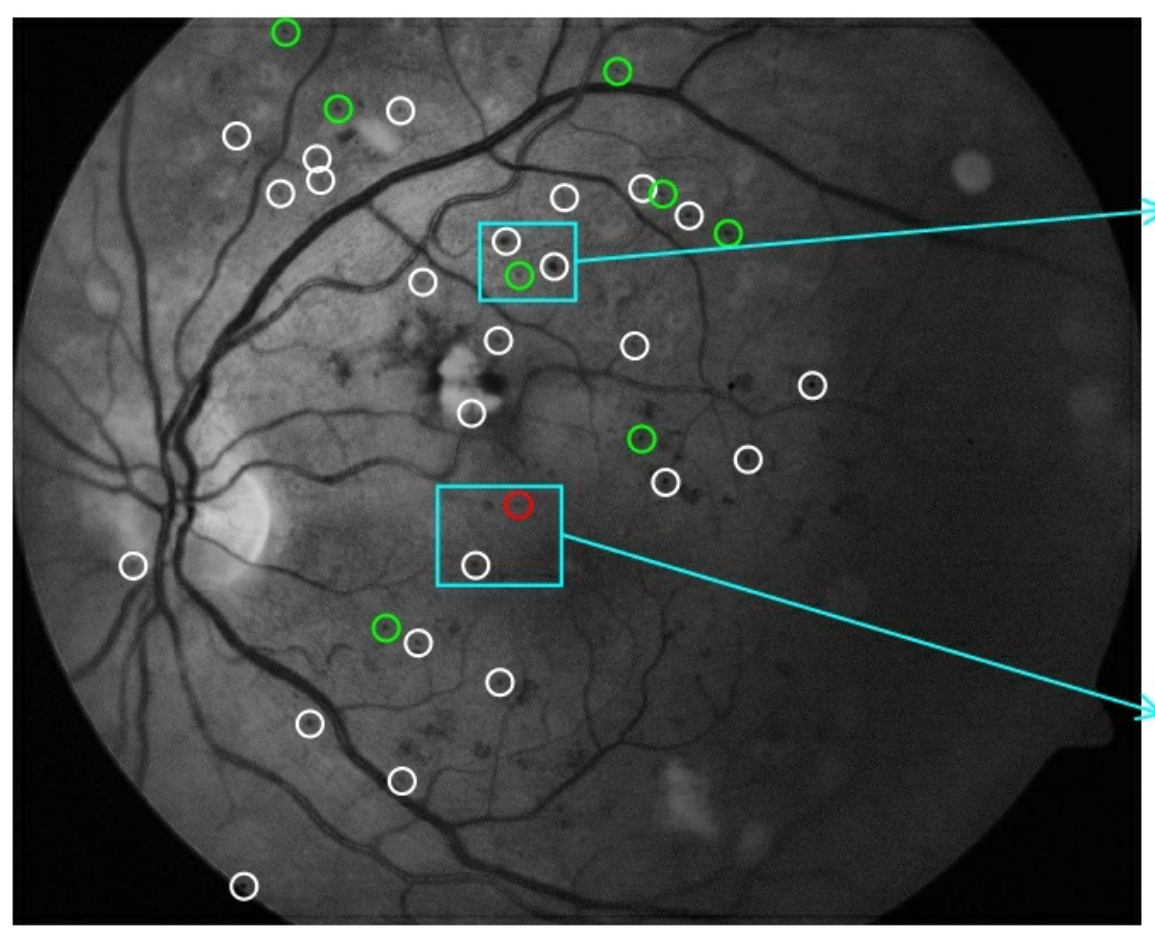

(a)

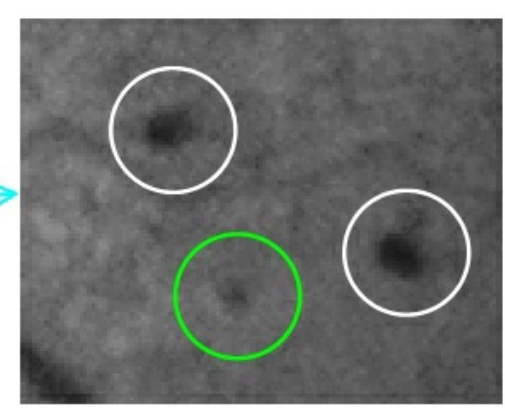

(b)

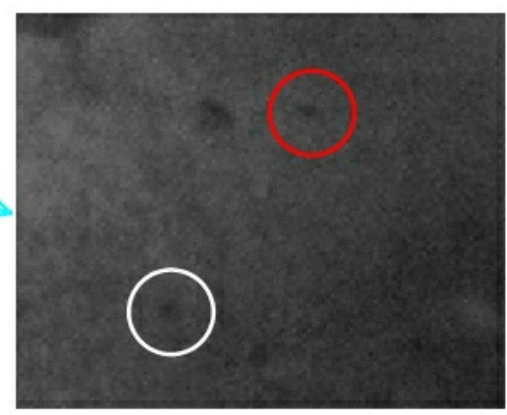

(c)

Figure 6

Lesion level evaluation for MA detection results on DIARETDB1 database. (a) Results of MA detection, where green circles indicate TPs, white circles indicate FPs, and the red circle indicates FN; (b) Examples of TP and FP; (c) Examples of FP and FN 


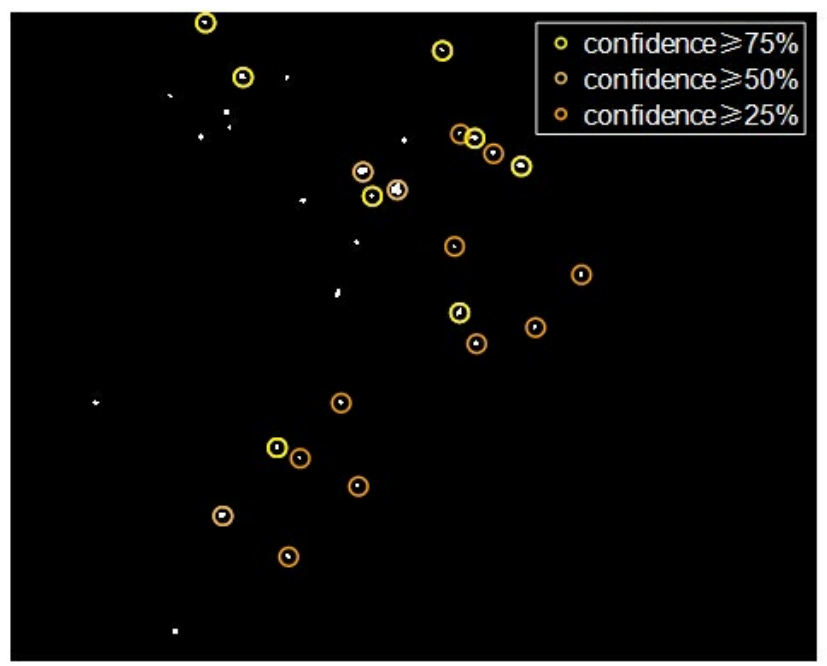

(a)

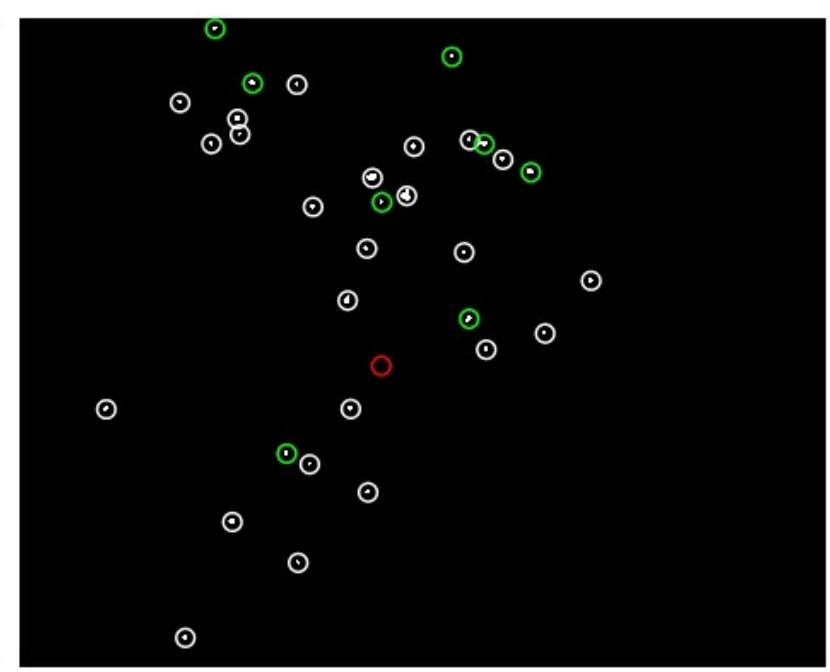

(b)

Figure 7

Analysis of MA detection results compared with ground truth on DIARETDB1 database. (a) MA detection results corresponding to dierent labeling condences, where yellow circles indicate labels with condence $75 \%$, orange circles indicate labels with condence $50 \%$, and brown circles indicate labels with condence $25 \%$. (b) Evaluarion of MA detection results with ground truth of condence $75 \%$, where green circles indicate TPs, white circles indicate FPs, and the red circle indicates FN, corresponding to Fig. 6

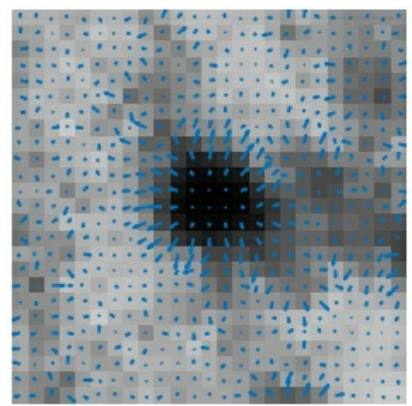

(a)

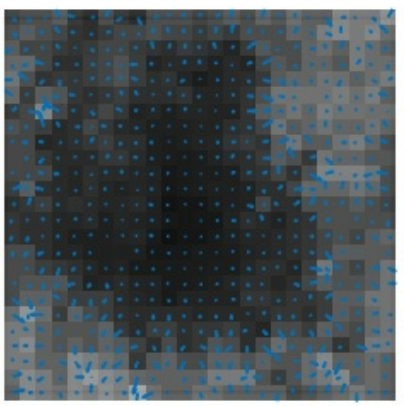

(b)

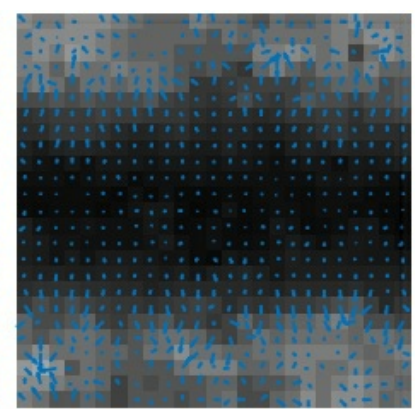

(c)

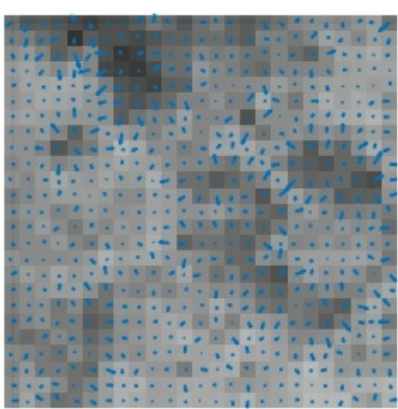

(d)

Figure 8

Comparison of dierent structures in 25*25 patch. (a) MA; (b) HM; (c) BV; (d) Background 


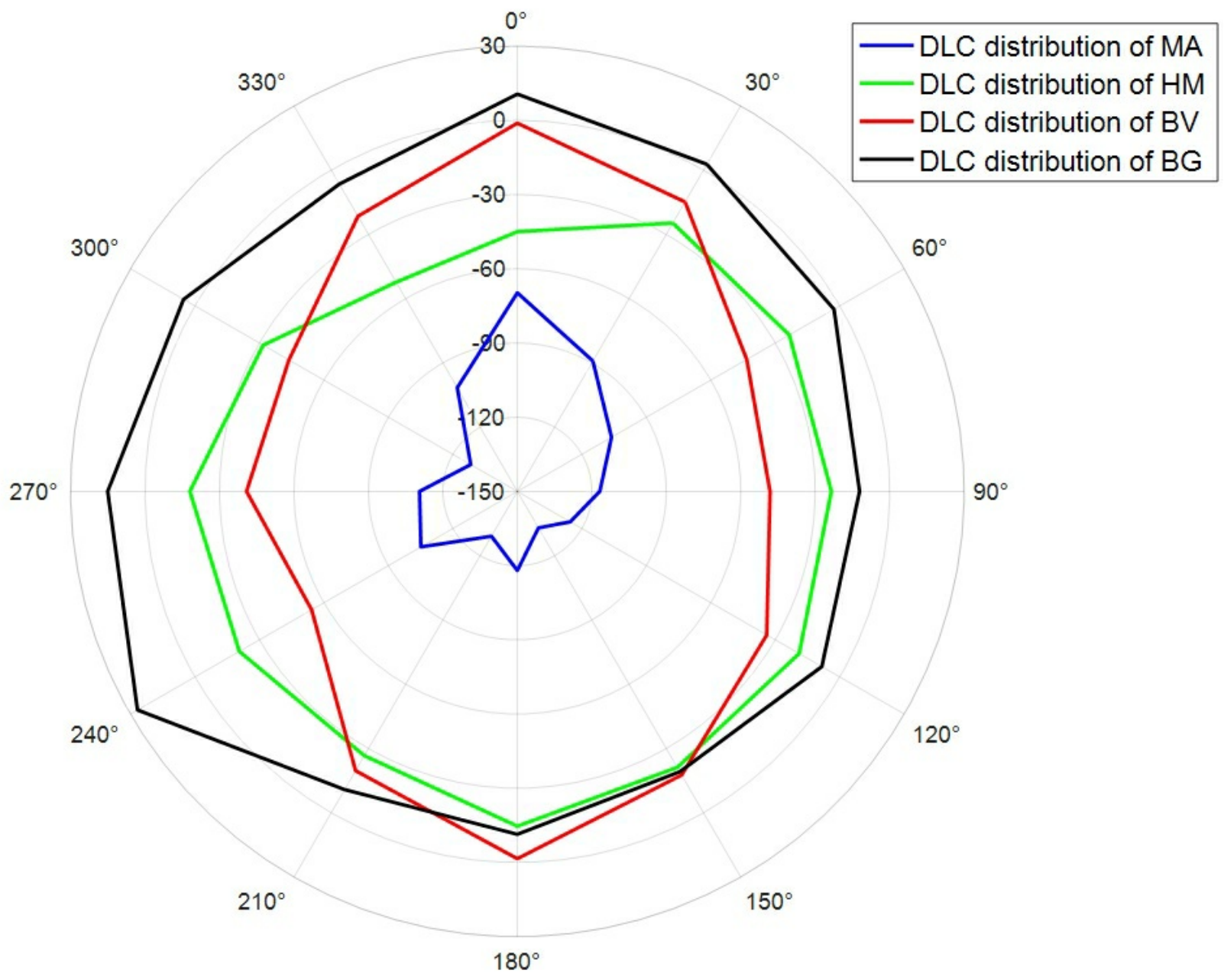

Figure 9

DLC distribution on dierent structures shown in Fig. 8, where radius indicates the DLC value along the direction angle 


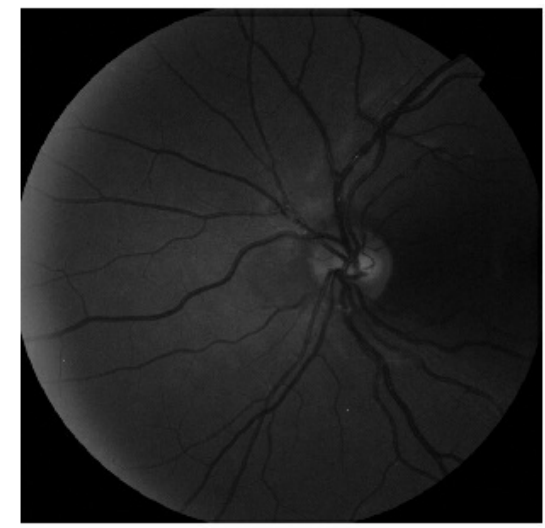

(a)

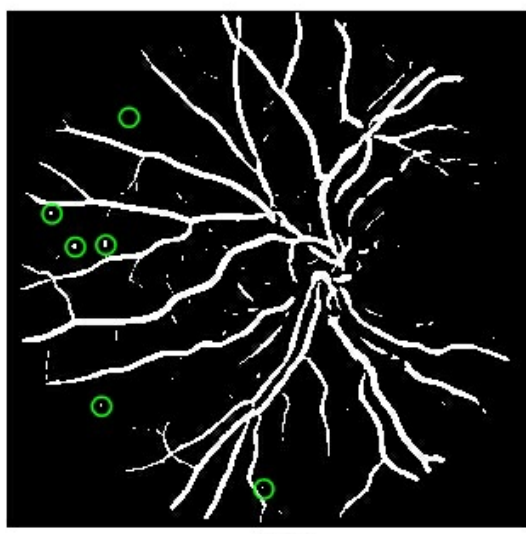

(d)

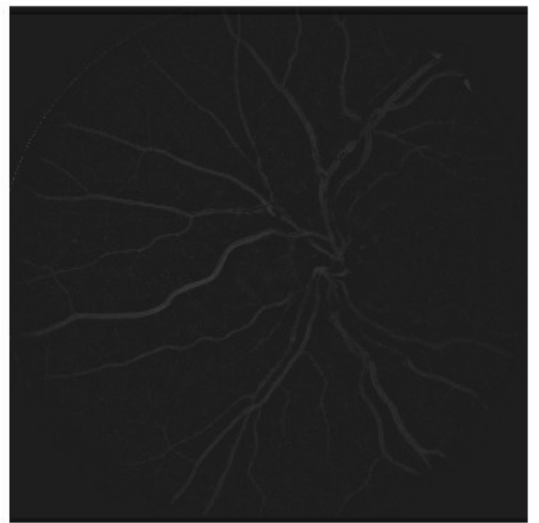

(b)

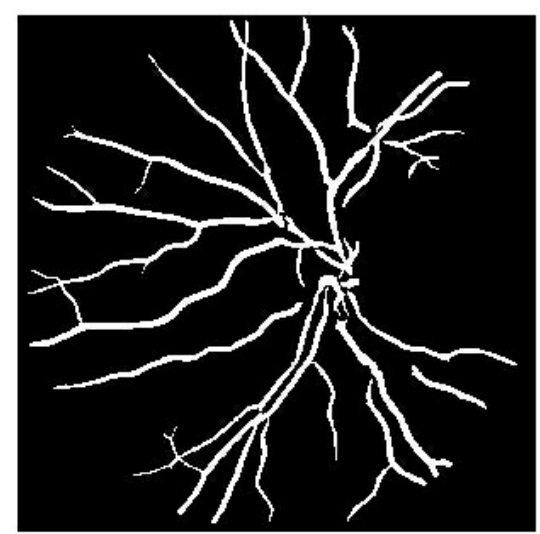

(e)

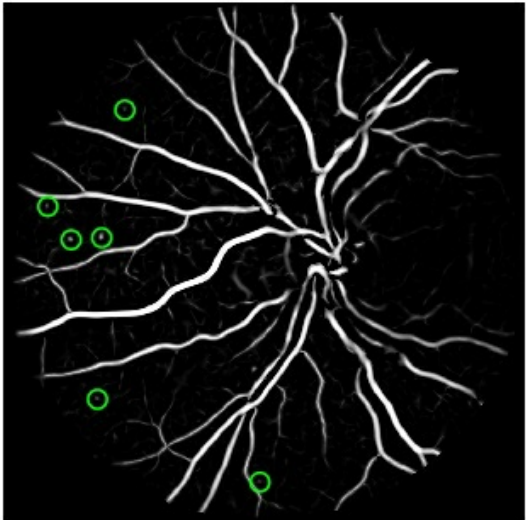

(c)

Figure 10

Process of blood vessels segmentation. (a) Green channel image; (b) Result of preprocessing; (c) BV enhanced image (green circles indicate MAs); (d) Preliminary BV segmentation (green circles indicate MAs); (e) Final result of BV segmentation 


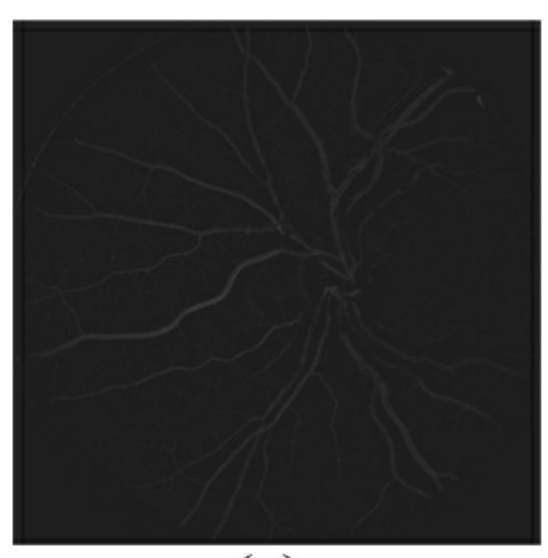

(a)

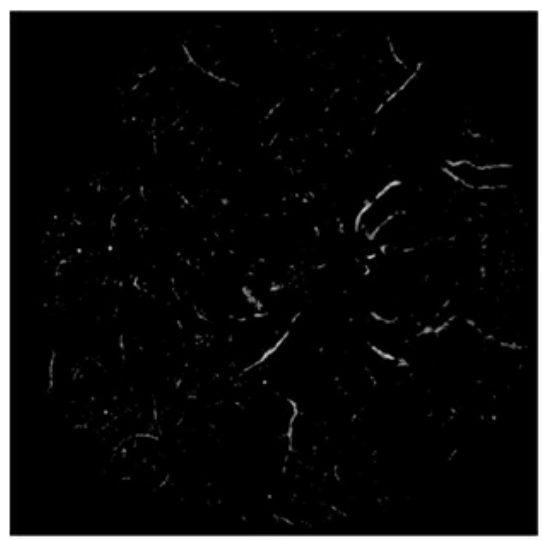

(d)

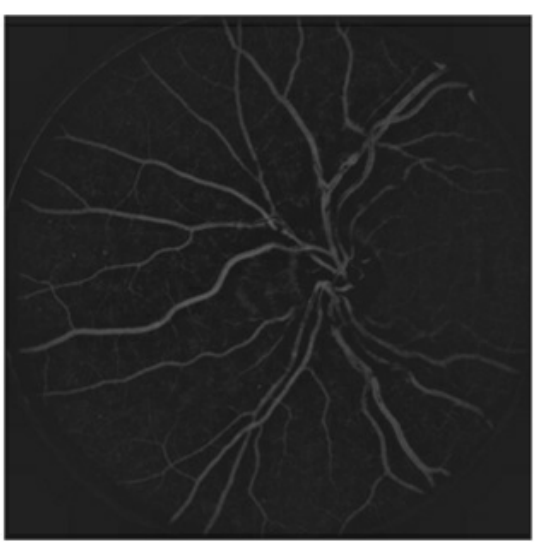

(b)

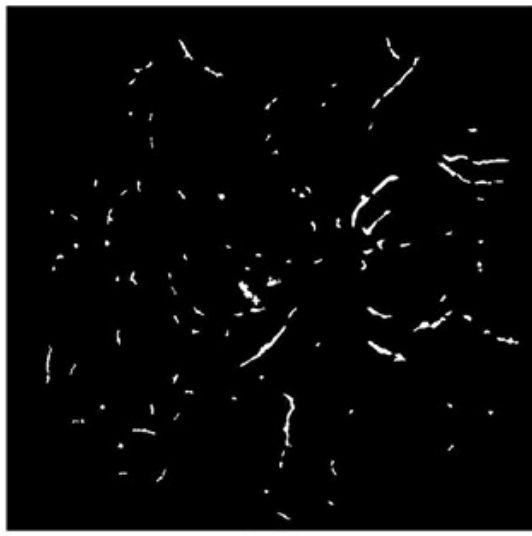

(e)

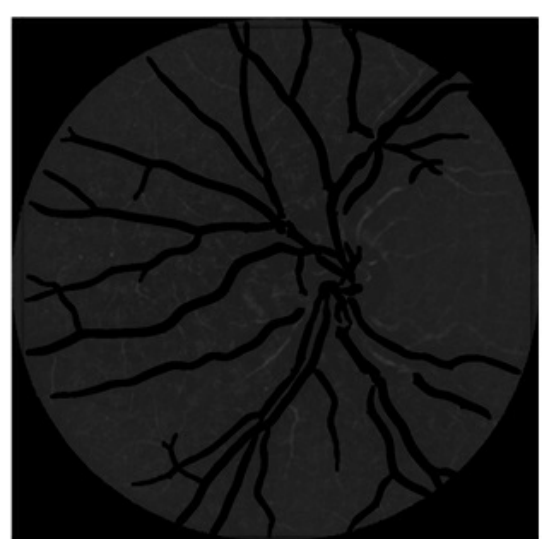

(c)

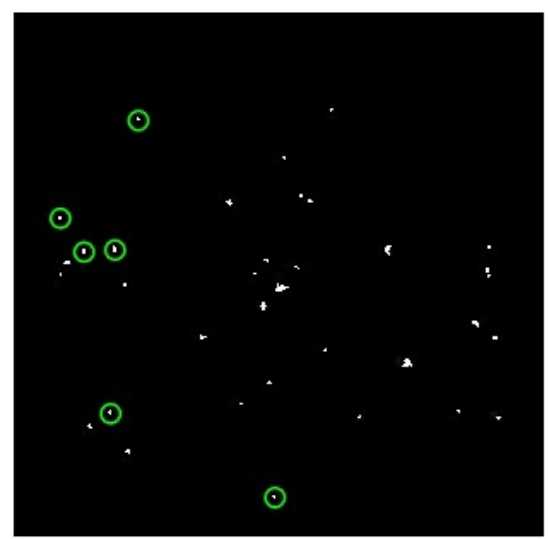

(f)

Figure 11

Process of MA candidate regions extraction. (a) Result of preprocessing before BV segmentation; (b) Result of further preprocessing; (c) Result of BV removal; (d) Result of contrast stretch; (e) Preliminary MA candidate regions; (f) Final result of MA candidate regions (green circles indicate ground truth of MAs) 


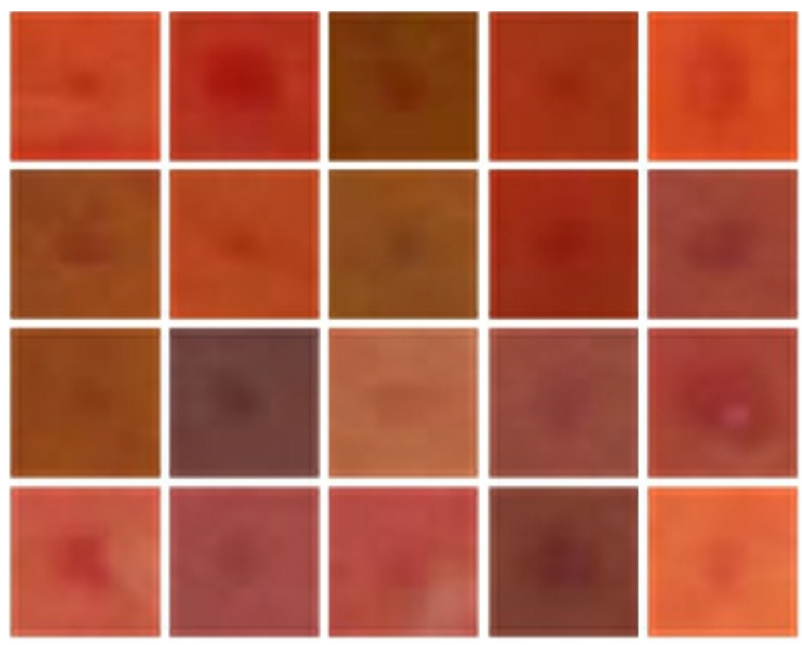

(a)
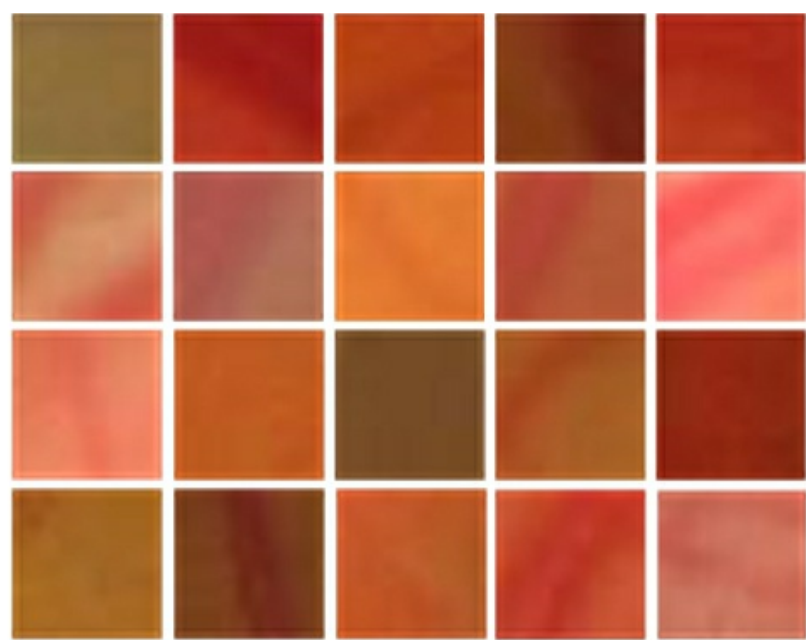

(b)

Figure 12

Examples of patches. (a) MA patches; (b) Non-MA patches 